\title{
Differential changes in hippocampal CaMKII and GluAl activity after memory training involving different levels of adaptive forgetting
}

\author{
Nicolas Fraize, ${ }^{1,2,3,5}$ Al Mahdy Hamieh, ${ }^{1,2,3,5}$ Mickaël Antoine Joseph, 1,2,3 \\ Monique Touret, ${ }^{2,3,4}$ Régis Parmentier, ${ }^{1,2,3}$ Paul Antoine Salin, ${ }^{1,2,3,6}$ \\ and Gaël Malleret ${ }^{1,2,3,6}$
}

${ }^{1}$ Forgetting and Cortical Dynamics Team, Lyon Neuroscience Research Center (CRNL), University Lyon 1, 69007 Lyon, France; ${ }^{2}$ Centre National de la Recherche Scientifique (CNRS), Unité Mixte de Recherche 5292, 69007 Lyon, France; ${ }^{3}$ Institut National de la Santé et de la Recherche Médicale (INSERM), Unité 1028, 69007 Lyon, France; ${ }^{4}$ Neurooncology and Neuroinflammation team, Lyon

Neuroscience Research Center (CRNL), 69007 Lyon, France

\begin{abstract}
Phosphorylation of CaMKII and AMPA receptor GluAl subunit has been shown to play a major role in hippocampal-dependent long-term/reference memory (RM) and in the expression of long-term synaptic potentiation (LTP). In contrast, it has been proposed that dephosphorylation of these proteins could be involved in the opposite phenomenon of hippocampal long-term synaptic depression (LTD) and in adaptive forgetting. Adaptive forgetting allows interfering old memories to be forgotten to give new ones the opportunity to be stored in memory, and in particular in short-term/working memory (WM) that was shown to be very sensitive to proactive interference. To determine the role of CaMKII and GluAl in adaptive forgetting, we adopted a comparative approach to assess the relative quantity and phosphorylation state of these proteins in the brain of rats trained in one of three radial maze paradigms: a RM task, a WM task involving a high level of adaptive forgetting, or a WM involving a low level of adaptive forgetting. Surprisingly, Western blot analyses revealed that training in a WM task involving a high level of adaptive forgetting specifically increased the expression of AMPA receptor GluAl subunit and the activity of CaMKII in the dentate gyrus. These results highlight that WM with proactive interference involves mechanisms of synaptic plasticity selectively in the dentate gyrus.
\end{abstract}

[Supplemental material is available for this article.]

Hippocampal-dependent memory formation is thought to require changes in synaptic efficacy (Bliss and Lomo 1973; Bliss and Collingridge 1993; Kandel 2001). The two major kinds of hippocampal-based synaptic plasticity are long-term potentiation (LTP) and long-term depression (LTD) (Bear and Malenka 1994; Bear and Abraham 1996). LTP can be artificially induced by application of brief trains of high-frequency stimulation and corresponds to an increase in synaptic transmission dependent on the phosphorylation state of key proteins such as glutamatergic $\mathrm{N}$-methyl-D-aspartate (NMDA) receptors and $\alpha$-amino-3-hydroxy-5-methyl-4-isoxazole propionic acid (AMPA) receptors subunits. NMDA receptors are ionotropic $\mathrm{Na}^{+} / \mathrm{Ca}^{2+}$ channels that have the property of being activated by glutamate release only if the postsynaptic membrane is depolarized (Elgersma and Silva 1999). Under these conditions, the $\mathrm{Mg}^{2+}$ ion that blocks the NMDA receptor channel when the membrane is at resting potential is released, liberating the channel and promoting a flux of $\mathrm{Ca}^{2+}$ through it (Herron et al. 1986). Consequently, during the induction of LTP, a rapid rise in postsynaptic intracellular $\mathrm{Ca}^{2+}$ concentration occurs in the postsynaptic compartment. This increase in $\mathrm{Ca}^{2+}$ leads to the activation of $\mathrm{Ca}^{2+} /$ calmodulin-dependent protein kinase II (CaMKII). Once activated, CaMKII that is local-

\footnotetext{
${ }^{5}$ These two authors contributed equally to this work.

Joint senior authors.

Corresponding author: gaelmalleret@gmail.com

Article is online at http://www.learnmem.org/cgi/doi/10.1101//m.043505.116.
}

ized predominantly in the postsynaptic density (PSD) can become autonomously and persistently active by autophosphorylation, thus acting as a synaptic tag driving new proteins to the activated synapse and contributing to a local and lasting increase in synaptic transmission. CaMKII autophosphorylation occurs at the Thr286 site of the $\alpha$ subunit (Fukunaga et al. 1995; Barria et al. 1997a). Once this site phosphorylated, the enzyme can phosphorylate itself even in the absence of $\mathrm{Ca}^{2+}$ (Miller and Kennedy 1986). Consistent with this hypothesis, genetic deletion of Thr286 has been shown to block LTP and memory (Giese et al. 1998) and noncompetitive inhibition of CaMKII during the maintenance phase of LTP has been shown to reverse LTP (Sanhueza et al. 2007). There is thus considerable evidence demonstrating that CaMKII is critical for LTP. Once activated, CaMKII phosphorylates AMPA receptors GluA1 subunits at their Ser831 sites. Such phosphorylation allows the integration of new AMPA receptors at the PSD (Barria et al. 1997b), further potentiating synaptic transmission. Recently however, CaMKII was also shown to interact with Arc/ Arg3.1 gene product to produce "inverse" synaptic tagging of inactive synapse (Okuno et al. 2012). By this process, CaMKII could

\footnotetext{
C 2017 Fraize et al. This article is distributed exclusively by Cold Spring Harbor Laboratory Press for the first 12 months after the full-issue publication date (see http://learnmem.cshlp.org/site/misc/terms.xhtml). After 12 months, it is available under a Creative Commons License (AttributionNonCommercial 4.0 International), as described at http://creativecommons. org/licenses/by-nc/4.0/.
} 
lead to LTD by promoting AMPA receptors endocytosis, thus preventing undesired enhancement of weak synapse in potentiated neurons.

Unlike LTP, LTD corresponds to a prolonged decrease in synaptic transmission. LTD is generally induced by low-frequency stimulation protocols and involves protein phosphatasedependent dephosphorylation processes of AMPA receptors (Dudek and Bear 1992; Lee et al. 2000). Whereas hippocampaldependent memory studies suggest that the long-term storage of information requires LTP-like mechanisms (Kandel 2001; Malleret et al. 2001; Martin and Morris 2002), the role of LTD is less clear (Bear and Malenka 1994; Braunewell and ManahanVaughan 2001; Etkin et al. 2006). It has been proposed that LTD could increase the signal-to-noise ratio of a memory trace. In agreement with this notion, we found that LTD could function to weaken previous memory traces. We showed that inhibiting the expression of the protein phosphatase PP2A (Nicholls et al. 2008) in transgenic mice prevented the dephosphorylation and internalization of AMPA receptors, and in consequence the restricted expression of a NMDA-dependent form of LTD (while leaving intact other classical forms of synaptic plasticity such as LTP or depotentiation). This mouse model was therefore particularly suited to study the role of LTD. We found that these mice had profound impairment at forgetting previously stored information suggesting that LTD could function to weaken previous memory traces, thereby preventing those traces from interfering with newly encoded information when new conditions demand it as it is the case in a working memory (WM) task (Nicholls et al. 2008).

Memory is not unitary and a distinction is often made between long-term/reference memory (RM) and short-term/WM. Unlike the well-known long-term memory, WM represents the short-term acquisition of trial-unique information (Baddeley 1981; Cowan 2008). It has been proposed that WM is thought to be a short-term form of a memory that, once used, is better to be forgotten or ignored in order not to interfere with later learning (Dudchenko 2004; Delaney and Sahakyan 2007). A consequence of this view is that forgetting would be required for WM. However, unlike memory, forgetting processes and their biological bases are poorly understood, probably because forgetting has often been seen as simply a lack of memory, a failed process that happens to us involuntarily (Levy et al. 2010; Sala 2010). Human studies, using directed forgetting, suggest just the opposite and propose that forgetting is equally important to memory and that some forms of forgetting are adaptive and essential to secure optimal storage of information (Rauchs et al. 2011). For instance, a waiter taking many similar orders during a shift would find forgetting of previous orders essential for the storage of relevant (e.g., new orders) information (Roberts and Dale 1981; Anderson et al. 1996; Rosenzweig et al. 2002; Dudchenko 2004). Examples of this adaptive role of forgetting such as these are numerous, but surprisingly we hardly know anything of its cellular or molecular underpinnings. In an attempt to determine the biological bases of such forgetting, we recently tested different groups of rats in an eight-arm radial maze in tasks involving reference memory (RM) or the processing of different levels of proactive interference (PI) (i.e., requiring different levels of adaptive forgetting of previous information) in WM (Joseph et al. 2015). Using the Immediate Early Genes zif268 and c-Fos as markers of neuronal activity, we found that many brain structures are activated by RM or WM processes involving or not forgetting, except the dentate gyrus (DG) of the dorsal hippocampus that remains silent when forgetting is needed. Conversely, we showed that inactivating the DG promotes forgetting. We therefore wanted to determine whether different plasticity changes were at work in the dorsal hippocampus, and in particular in the DG, after training in RM or WM tasks involving, or not involving adaptive forgetting. To answer this question, we tested a new group of rats in the same behavioral tasks and assessed the expression and phosphorylation state of molecular markers of synaptic plasticity such as CaMKII (P-CaMKII) and glutamate AMPA receptor subunit GluA1 in the DG as well as in the CA1 and CA3 region of the dorsal hippocampus, but also the prefrontal cortex (PFC), another region greatly involved in memory processing (Botreau et al. 2004; Maviel et al. 2004; Kesner and Churchwell 2011). The results of this study are described below.

\section{Results}

\section{Behavior}

Rats were trained as previously described (Joseph et al. 2015) (see also Materials and Methods and Fig. 1A-E). The performance of rats trained in the three different tasks (RM, LIWM, and HIWM), are represented in Figure 1F. ANOVAs revealed a significant Group effect $\left[F_{(2,30)}=57.472 ; P<0.0001\right]$, a significant Block effect $\left[F_{(4,120)}=9.317 P<0.0001\right]$, as well as a significant Group $\times$ Block interaction $\left[F_{(8,120)}=13.762 P<0.0001\right]$. Post hoc analyses revealed that RM rats significantly improved their performance over time $\left[F_{(9,36)}=18.413 ; P<0.0001\right]$ and reached $85 \%$ correct choices on the last block of days, indicating a learning of the general rules and strategies required to solve the task. In WM groups, we confirmed earlier findings showing that proactive interference (PI) affect WM performance (Joseph et al. 2015). At the beginning of training, both LIWM and HIWM groups started at almost $90 \%$ of correct choices. LIWM rats kept high scores throughout the entire experiment, slightly increasing with time and reaching 100\% on Block 5 (no significant Block effect). On the contrary, rats trained in the HIWM task showed a decrease in performance over days indicating that accumulation of PI critically distorts WM performance with time (significant difference in score was shown on the last two blocks of days between LIWM and HIWM $P=0.0055$ for block 4 and $P=0.0005$ for block 5) (Fig. 1F).

\section{Western blot assays}

After $10 \mathrm{~d}$ of training, rats were sacrificed and we carried out Western blot assays of the molecular markers of synaptic plasticity CaMKII and AMPA receptor GluA1 subunit. We also quantified the level of NMDA receptor subunits GluN1, GluN2A, and GluN2B. The Western blot analysis was carried out in the CA1 and CA3 region of the dorsal hippocampus as well as in the DG and the PFC.

\section{GluAl phosphorylation ratio is modulated in the DG after HIWM training}

First, we quantified the total form of different subunits constituting AMPA and NMDA receptors within both the PFC and the dorsal hippocampus. No changes in the overall expression of NMDA receptors subunits were observed when compared with control rats after training in any of the three behavioral tasks and whatever the studied brain structure (Fig. 2A-C; Supplemental Tables $1-$ 5). Our results revealed no changes in GluA1 expression in any of the groups and/or studied structures (see Fig. 2D; Supplemental Tables 1-5), except for a $30 \%$ increase observed specifically in the DG after HIWM training when compared with the control group $(P=0.0005)$. We also assessed the phosphorylation state of GluA1. We showed that the phosphorylation ratio of the GluA1 subunits at the ser831 (ratio ser831/total GluA1) and ser845 (ratio ser845/total GluA1) sites did not differ from the control group after training in anyone of the behavioral conditions in CA1, CA3, or the PFC (Supplemental Fig. S1; Supplemental Tables $1-5)$. In the DG and after RM training, we did not observe any 
A

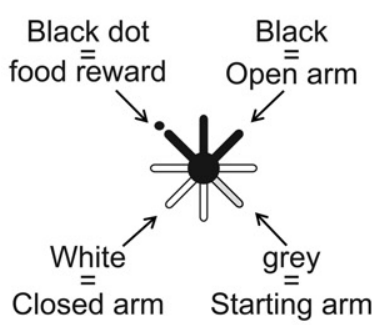

B

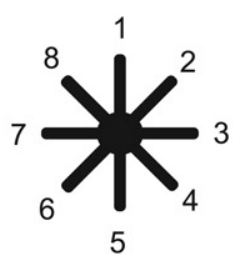

C

Reference Memory task (RM)

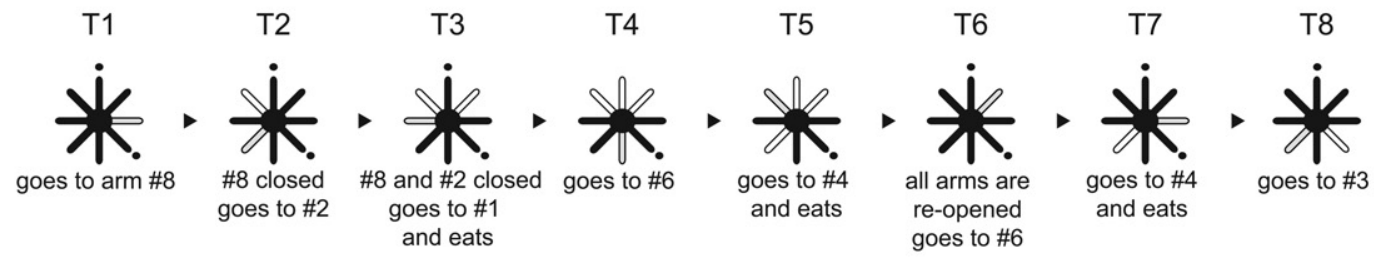

D

Low Interference Working Memory task (LIWM)

T1

$\mathrm{T} 2$

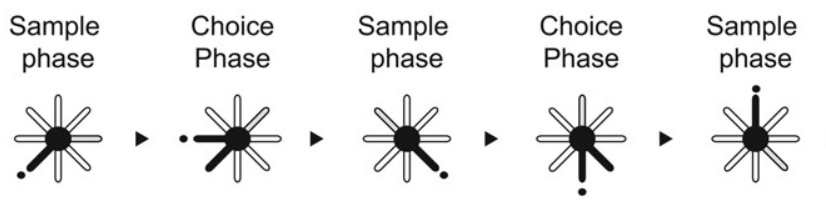

T3

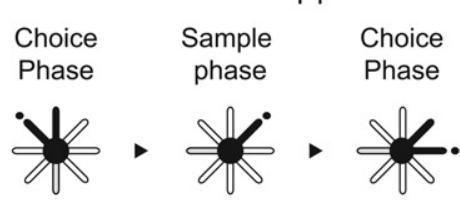

E

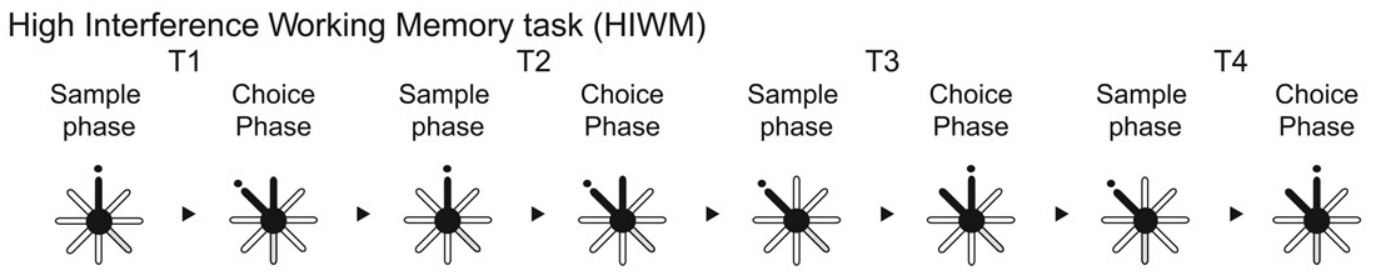

$\mathbf{F}$

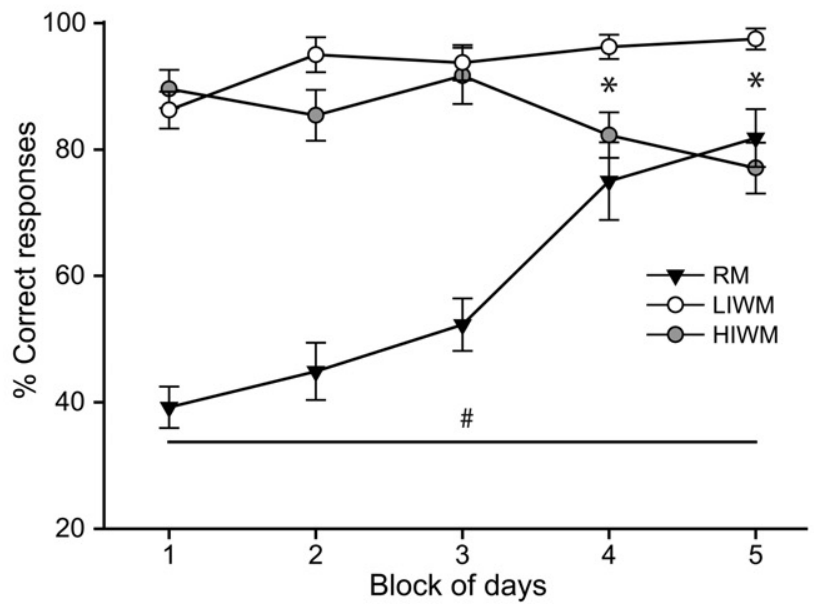

Figure 1. Proactive interference impairs the performance of rats trained in the HIWM task. $(A, B)$ schematic representations of the maze with the arms' attributed numbers and legends. ( $C-E$ ) Behavioral paradigms (see Materials and Methods for details) and schematic representation of one training day for each of the experimental groups. (C) Reference memory (RM) training. The same two arms (here \#1 and \#4, (B)) are baited every day for each trial. Each daily session consisted of eight trials $(\mathrm{T} 1-\mathrm{T} 8)$. (D) Low interference working memory (LIWM) training. Each day consisted of four trials (T1 - T4) of two phases each. ( $E$ ) High interference working memory (HIWM) training. Same as $D$ except that the same pair of arms is used every day for each trial. Consequently, the trials are very similar to each other and it is therefore necessary to ignore and forget previous trials to complete an ongoing trial. $(F)$ Behavioral performances of each group of rats. Percentage of correct choices \pm S.E.M per Block (each Block $=2 \mathrm{~d}$ of training) in RM, LIWM, and HIWM tasks. Black line represents the chance level for both WM tasks. * indicates significant difference between HIWM and LIWM groups $(P<0.05-$ ANOVA), \# indicates a significant block effect for the RM group $(P<0.0001)$. 
A
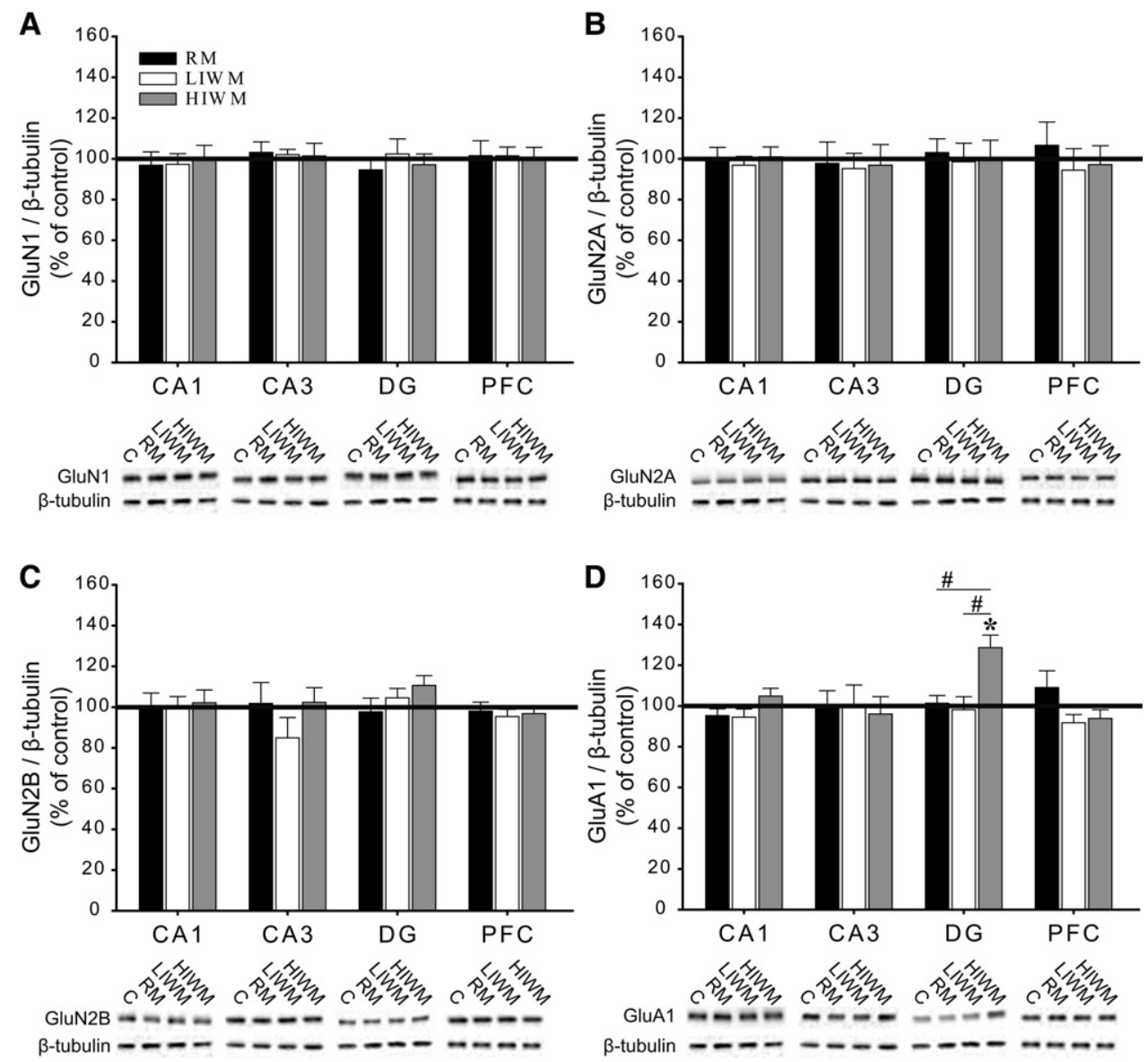

Figure 2. Western blot quantification of overall GluN2A, GluN2B, GluN1, and GluA1 expression. No change was observed in the overall expression of GluN (NMDA receptors) subunits $(A-C)$, but a significant increase in the overall GluA1 expression was observed after HIWM training and when compared with controls and LIWM and RM trained rats $(D)$. Data are expressed as mean \pm S.E.M.-Experimental group values are expressed as $100 \% \pm$ S.E.M. of average control group (black line). Multiple comparisons with Holm-Bonferroni's correction. ${ }^{*}$ indicates difference with the control group, \# indicates difference between groups $\left({ }^{*}, \#\right) P<0.01$.

change in the phosphorylation ratio at either phosphorylation site (for ser831 site $P=0.3953$; for ser845 site $P=0.7807$ ). However, we observed a significant decrease in the DG of the ser845 phosphorylation ratio after LIWM training when compared with controls $(P=0.0072), \mathrm{RM}(P=0.0039)$, and HIWM $(P=0.0015)$ trained rats (Fig. 3; Supplemental Tables 1, 4). No other significant differences between groups were observed although a nonsignificant increase in the phosphorylation ratio of both ser831 and ser845 could be seen after HIWM training when compared with RM training (Fig. 3; Supplemental Tables 1, 4).

\section{HIWM training induces an increase in CaMKII activation}

We also assessed the total quantity of CaMKII after training in the radial maze. We failed to observe significant changes after any of the training conditions when compared with the control group (Supplemental Fig. S2; Supplemental Tables 1-5). We then assessed the level of CaMKII activated form by quantifying the phosphorylation ratio of CaMKII (P-CaMKII/total-CaMKII) and observed, in the DG, a large increase of $160 \%$ (when compared with controls) of CaMKII activated form after HIWM training $(P=0.0046)$. A nonsignificant decrease may also be observed after RM training when compared with controls (Fig. 4; Supplemental Tables 1,4$)$, even if this decrease was found significant when compared with HIWM trained rats $(P=0.0092)$. In contrast, in CA1, we observed a nonsignificant increase of CaMKII activated form when compared with controls after RM training, but also a trend (not significant) toward a decrease of CaMKII activity after LIWM training. We did not observe any other changes in P-CaMKII in any other group or for any other structure.

\section{CaMKII activation in the DG is correlated with GluAl phosphorylation}

We wanted to confirm that the large increase in CaMKII activity observed in the DG after HIWM training might be indeed related to synaptic plastic changes. We thus assessed for each subject, and in the DG, the correlation between the phosphorylation ratio of GluA1 (ser831 and ser845) and the phosphorylation of CaMKII. We found a positive correlation between Ser831 phosphorylation and CaMKII activity specifically after HIWM training $(P=$ 0.0209-see Fig. 4C) confirming the key role of CaMKII in the phosphorylation of GluA1 at the ser831 site. No other significant correlation was observed between GluA1 phosphorylation and CaMKII activity (after RM training, for ser831 phosphorylation versus CaMKII activity: $P=0.7528$, for ser845 phosphorylation versus CaMKII activity: $P=0.7414$. After LIWM training, for ser831 phosphorylation versus CaMKII activity: $P=0.0965$, for ser845 phosphorylation versus CaMKII activation: $P=0.2554$. After HIWM training, for ser845 phosphorylation versus CaMKII activity: $P=0.2568$ ). 
A

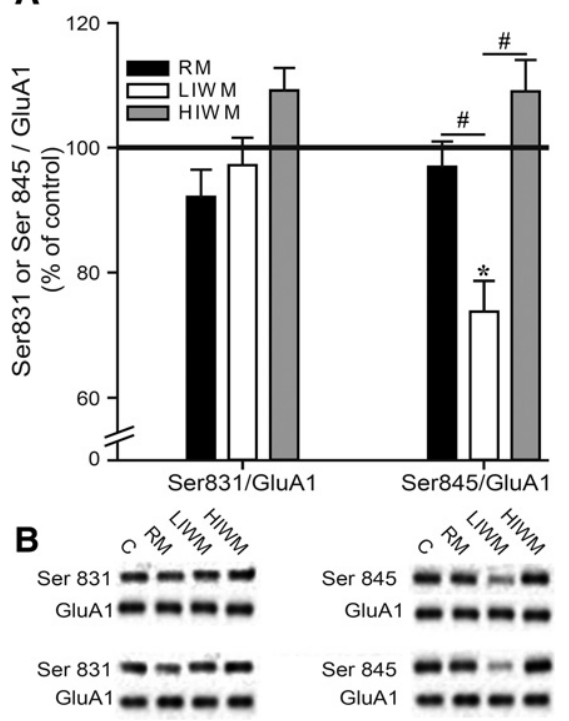

Figure 3. Western blot quantification of the phosphorylation ratio of GluA1 in the DG. (A) After LIWM training, Ser845 phosphorylation ratio is decreased when compared with controls and when compared with the HIWM group. Ser831 phosphorylation seems to be increased in the HIWM group when compared with the LIWM group but no significant differences by Mann-Whitney U-tests were observed after HolmBonferroni's corrections for multiple comparisons. Data are expressed as mean \pm S.E.M. - Experimental group values are expressed as $100 \% \pm$ S.E.M. of average control group (black line). Multiple comparisons with Holm-Bonferroni's correction. ${ }^{*}$ indicates significant difference compared with the control group: $P<0.01$. \# indicates significant differences between experimental groups: $P<0.01$. (B) Representative immunoblots of the gels are shown.

\section{Discussion}

With this study, we showed that behavioral training involving the long-term or short-term storage of information and/or different levels of adaptive forgetting differentially affect the expression and phosphorylation state of biological markers of synaptic plasticity in the three main areas of the dorsal hippocampus. Our Western blot analysis revealed that the processing of interference in WM might involve specific synaptic plasticity changes in the DG. These changes involve an increase in the expression of AMPA receptor GluA1 subunit and in the phosphorylated/active state of CaMKII at the Thr286 site of the $\alpha$ subunit.

Different studies have suggested that hippocampal LTP — that underlies the long-term storage of information in RM-requires a sustained increase in the number of AMPA receptors at the synaptic level (Nayak et al. 1998; Shi et al. 1999; Dong et al. 2015). Consequently, our finding that RM training does not induce any change in GluA1 expression may seem surprising. This may be due to the fact that we did not isolate membranous (postsynaptic) fraction from our hippocampal lysates. Changes in the number of receptors at the PSD are an important mechanism underlying LTP and long-term memory (Whitlock et al. 2006). Such changes may occur at the postsynaptic sites after RM training but may be invisible in our total hippocampal lysates. However, to isolate membranous fraction requires an important quantity of biological sample. The fact that we were particularly interested in changes at play in the DG based on our earlier findings (Joseph et al. 2015) led us to micro-punch our samples to isolate the three different hippocampal subregions. Unfortunately, this manipulation greatly reduced the quantity of available biological material and thus prevented the isolation of the synaptic fraction from our hippocampal lysates. In consequence, we cannot rule out the hypothesis that a GluA1 increase might have been observed in membranous fractions of our samples after RM training. However, the fact that RM training does not induce any change in GluA1 expression may not be surprising as some studies have shown that GluA1 knockout mice displayed a specific and restrictive deficit in WM but not RM (Reisel et al. 2002; Schmitt et al. 2003; Sanderson et al. 2009). In fact, some authors have shown that this genetic deletion of GluA1 may even increase RM (Schmitt et al. 2003) by reducing the detrimental effects of shortterm memory on subsequent long-term learning (Sanderson et al. 2009). Alternatively, the absence of GluA1 expression in our hippocampal lysates after RM training may also be due to the fact that rats have been sacrificed after $10 \mathrm{~d}$ of training, a time when the acquisition of the RM task rules has already been achieved (as shown by the plateaued performance). This may explain why no change in GluA1 expression has been observed after $10 \mathrm{~d}$ of RM training.

Unlike the RM task, the LIWM task always (up to the last days of training) requires the short-term storage of information. In this delayed-non-match-to-place task, different pairs of arms are used for each trial. The deleterious action of PI is thus negligible in this paradigm (as reflected by the behavioral performance superior on the last days of LIWM training when compared with HIWM training). We found that LIWM training induces a decrease in GluA1 phosphorylation at the ser845 site in the DG. Dephosphorylation of ser845 has been described as a signal for GluA1 internalization and lysosomal degradation of AMPA receptors leading to LTD (Fernandez-Monreal et al. 2012). Our results thus suggest that when the interference load is not strong enough to induce a drop in behavioral performances (as it is the case in HIWM), LTD processes may occur to erase memories of the trials that have been performed in the past and that are no longer useful. After HIWM training on the other hand, when the load of interference is too high to be dealt with efficiently (and when a decrease in performance is observed), the level of phosphorylation of these GluA1 subunits in the DG of HIWM trained rats was found to be unchanged when compared with controls level. We may thus hypothesize that the increase in GluA1 expression that we observed after HIWM training reflects changes in cytosolic stores of AMPA receptors, but may not be a sign of an increased integration of new AMPA receptors at the synaptic level. These receptors may just be massively available in the DG granule cells of animals trained to be extremely flexible in their information processing as this HIWM task requires both the short-term storage of information, but also forgetting of previous trials to counteract the deleterious impact of PI on the storage of newer information. This extreme cognitive flexibility could require fast relocation of these receptors from the cytosol to the PSD for rapid memory storage, and from the PSD to the cytosol for fast forgetting once this information becomes irrelevant. HIWM training could thus require both the recruitment and internalization of functional AMPA receptors at the PSD level. It has been shown that blocking hippocampal AMPA receptors-dependent LTP could impair WM (Reisel et al. 2002; Schmitt et al. 2005). In contrast, our previous work showed that the inhibition of dephosphorylation and LTD processes impaired the processing of interference in WM (Nicholls et al. 2008). Altogether, these results suggest that WM may rely on both hippocampal synaptic potentiation and depression. Indeed, it is worth noting that the level of phosphorylation of GluA1 subunits (ser845), even if not increased when compared with the yoked control group, was in fact increased in the DG of HIWM rats when compared with rats trained in the LIWM tasks suggesting that the processing of PI could in fact require the phosphorylation of AMPA receptors, and therefore synaptic potentiation. This increase in the phosphorylation of GluA1 in the DG was also correlated to CaMKII activity. In fact, CaMKII activity was 

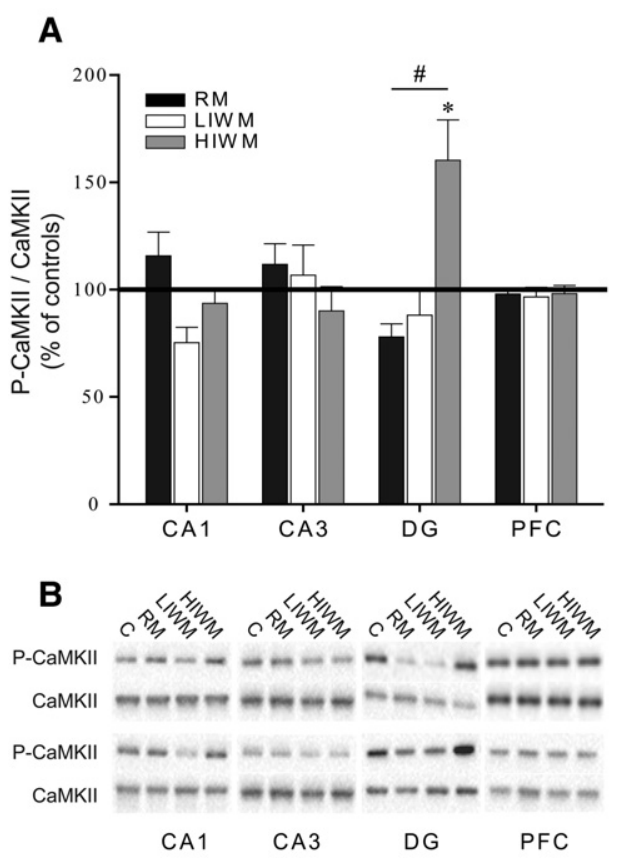
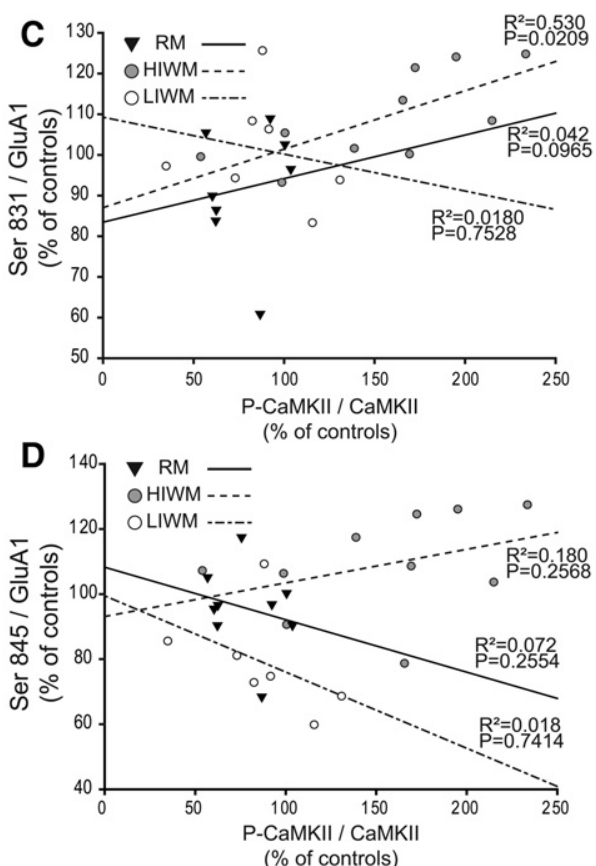

(\% of controls)

Figure 4. CaMKII is strongly activated after HIWM training. (A) Western blot quantification of the phosphorylation ratio of CaMKII (P-CaMKII/CaMKII). After RM training, phosphorylation of CaMKII seems to be increased in CA1, but decreased in the DG when compared with controls. However, no significant differences by Mann-Whitney U-tests were observed after Holm-Bonferroni's corrections for multiple comparisons in this structure. In contrast, after HIWM training, CaMKII phosphorylation is strongly increased in the DG. Data are expressed as mean \pm S.E.M., experimental group values are expressed as $100 \% \pm$ S.E.M. of average Control group (black line). Multiple comparisons with Holm-Bonferroni's correction. ${ }^{*}$ Indicates significant difference compared to the control group: $P<0.01$. \# indicates significant differences between experimental groups: $P<0.01$. (B) Representative immunoblots and quantification of the gels are shown. (C,D) correlation between CaMKII activity (phosphorylation ratio) and GluA1 phosphorylation at the ser831 (C) and the ser845 $(D)$ sites in the DG. Data are expressed as normalized ratio (percentage of control) $\left(^{*}\right) P<0.05$; (Spearman's correlation test).

massively increased in the DG of rats trained in the HIWM task. As mentioned above, CaMKII has long been shown to be involved in LTP and long-term memory storage (for review, see Lisman et al. 2012), and we here showed that rats trained in the long-term/ RM paradigm did express an elevated, albeit not significant, amount of activated (phosphorylated) CaMKII in the CA1 area of the dorsal hippocampus. However, studies on transgenic mice have also shown that excessive CaMKII activity can bidirectionally alter synaptic plasticity, facilitating LTP in response to 10 or 100 $\mathrm{Hz}$ stimulation but also increasing LTD in response to $3 \mathrm{~Hz}$ stimulation (Wang et al. 2003). It has been suggested that such abnormal LTD could be responsible for the aberrant and selective degradation of memory traces seen in these mice (Cao et al. 2008). The increase of CaMKII activity that we observed in the DG could thus reflect two processes. The first one implies that HIWM training could activate CaMKII-dependent phosphorylation mechanisms leading to LTP and consolidation of irrelevant information into long-term memory that may potentially be detrimental to the flexible use of memory in the HIWM task. This increase in synaptic potentiation and phosphorylation of CaMKII (but also GluA1) could thus be the cause of the decreased performance seen in this task at the end of training. One could postulate that this increase in phosphorylation of CaMKII (but also GluA1) could also be short-lived (Whitlock et al. 2006) and that, for forgetting purposes, synaptic potentiation and phosphorylation levels could go back to controls' level after training, possibly during sleep, a period favorable for synaptic downscaling (Tononi and Cirelli 2003). The other hypothesis implies that CaMKII increased activity could be beneficial to LTD, and therefore to the degradation (and thus adaptive forgetting) of irrelevant memory traces previously stored in memory during the HIWM task.
Further work should determine which of these two possible processes is involved in forgetting and the processing of PI. However, we here confirmed that the DG of the dorsal hippocampus is a crucial area involved in these processes. Our most significant results were found in this brain region for rats trained in the HIWM paradigm. This result is concordant with our previous study (Joseph et al. 2015) showing that (1) HIWM training was responsible for a very restricted and specific inactivation of Zif268 and c-Fos expression in the DG, and that (2) inactivating this structure was beneficial to the processing of interference. It is therefore not surprising to see important changes in the expression of synaptic plasticity markers in the DG after HIWM training. Our results can thus be explained as followed: the reduction of proactive interference could involve and require the inactivation of the DG and its pattern separation function, a result in agreement with previous findings showing that ablation of hippocampal neurogenesis (occurring in the DG) lead to improved processing of proactive interference (Saxe et al. 2007). However, a potential high degree of activation of a small neuronal population in the DG (as shown by a decreased Zif268 and c-Fos expression) in the HIWM task could also result in a maintained increase in synaptic plasticity (LTP) and in the storage of irrelevant information detrimental to HIWM training. Alternatively, the increase of synaptic plasticity in the DG could involve not synaptic potentiation, but synaptic depression that could participate to the inactivation of the DG required for forgetting and the processing of interference.

Our study also highlights interesting aspects on hippocampal function and physiology. It has been shown that CA3 may support the process by which a complete memory can be retrieved from only partial or degraded cues represented in this memory, a function called "pattern completion" (Gold and Kesner 2005) or 
auto-associative memory (Kesner and Rolls 2015). Altogether, our present and previous studies (Joseph et al. 2015) indicate that CA3 is activated during HIWM but that this activation does not require long-term form of plasticity, although synaptic plasticity changes seem to occur in CA3's afferent region (i.e., DG). It is thus possible that HIWM training could require plasticity changes in the DG to maintain the firing of CA3 neurons in a recurrent network that is termed an attractor state (Kesner and Rolls 2001). Interestingly, our results tend to show that a form of memory (i.e., RM) induces opposite changes in CaMKII activity in two different areas of the hippocampus (i.e., CA1 and DG) even if these changes could not be considered significant after correction for multiple comparisons. CA1 has long been shown to be implicated in the retrieval of spatial memory (Dillon et al. 2008). In line with these findings, CaMKII seems to be activated after RM training in CA1. In contrast, CaMKII seems to be inactivated in the DG after training in this task. Although these results are only correlational, one may hypothesize that the different substructures of the hippocampus could have antagonistic roles in memory processing.

\section{Materials and Methods}

\section{Subjects}

A total of 66 three-month-old Dark Agouti rats initially weighing 200-250 g at the beginning of the experiment were purchased from Harlan France. They were kept in a 12/12h light-dark cycle with ad libitum access to food and water but were subsequently food deprived and maintained at $85 \%$ of their free-feeding weight throughout the experiment. Rats were housed in cohorts of two such that each rat that was to acquire a high interference, low interference WM or RM task was housed with its yoked control. Therefore, three groups learned a radial maze task (high interference working memory (HIWM), low interference working memory (LIWM), and reference memory (RM) tasks), and three groups served as their respective controls (Yoked HIWM or YHIWM, Yoked LIWM or YLIWM, and Yoked RM or YRM). This study was carried out in strict accordance with the recommendations of the Lyon 1 University ethical committee for the use of experimental animals and of the European committee (2010/63/EU). The protocol was approved by the Lyon1 University ethical committee for the use of experimental animals (Permit Number: CE2A-UCBL 55). All efforts were made to minimize suffering.

\section{Behavioral apparatus}

An eight-arm radial maze requiring the use of spatial orientation and memory was used throughout the entire experiment for all tasks. This maze consisted of eight arms $(87 \mathrm{~cm}$ long $\times 12 \mathrm{~cm}$ wide) radiating from an octagonal central platform $(34 \mathrm{~cm}$ diameter). The entrance of each arm was blocked by opaque Perspex doors that could be automatically lowered (pneumatic system) by the experimenter located in a room directly adjacent to the testing room. Squared food wells of $2 \mathrm{~cm}$ and $0.5 \mathrm{~cm}$ deep were fixed at $0.5 \mathrm{~cm}$ beyond the end of maze arms. Food rewards (Dustless Precision Pellets; Bioserve) could be placed in these food wells. The maze was located in a room with a number of extramaze cues (e.g., poster, door, furniture). A video camera, connected to a video recorder and a monitor, was fixed above the maze. Behavior of the rats in the maze was videotaped for later examination.

\section{Behavioral protocol}

Food-deprived rats had to retrieve food rewards (sugar pellets, Bioserv) at the end of the maze's arms using spatial navigation and distal visual cues surrounding the maze. Rats underwent a 6-d habituation period during which they became accustomed to the radial maze environment and learned to find rewards in the arm wells. After habituation, they were pseudorandomly assigned to one of the following groups (see below and Joseph et al. 2015); whatever their group assignment, they were able to complete eight runs to an arm per day, making the three tasks strictly comparable in terms of motivational, emotional, and motor processes.

\section{Reference memory task}

Rats trained in the RM task ( $n=11$ ) had to retrieve food pellets in two arms of the maze. These two arms remain constant and were the same every day for the entire $10 \mathrm{~d}$ ( $=5$ Blocks of two daily sessions) of training (Bontempi et al. 1999). Rats were initially placed in a pseudorandomly chosen starting arm (gray arms, Fig. 1C) at which point all arms of the maze were opened (white arms, Fig. 1A). A transparent blocker prevented rats from going backward to the food well of the starting arm. Once a rat chose one of the arms (an arm selection was defined when the animal reached the arm's half way), the door to that arm was closed. After consuming the food reward in the case of a correct choice, or not in the case of an incorrect choice, rats were returned to a transfer cage adjacent to the maze for a short delay of $15 \mathrm{sec}$. The doors to previously chosen arms remained closed until both food reward were retrieved to prevent the rat to return into such arms (committing WM errors). After both food pellets were retrieved (for instance, on trial T5 in the example given in Fig. 1C), the two previously baited arms were rebaited and all arms were reopened (e.g., T6) (Fig. 1C). Rats underwent eight trials per day and the maximum score per day was fixed at eight pellets eaten. The latency to choose an arm as well as the number of correct choices was scored. Each experimental RM rat was paired with a YRM rat that performed the same amount of motor activity and ate the same number of pellets. These yoked controls $(n=11)$ were forced to enter into pseudorandomly chosen arms and were either reinforced or not depending on the performance of their experimental matched rat. The starting and destination arms varied between trials in such a way that yoked controls could not use motor memory to predict which direction they had to go. The use of yoked controls allows the experimenter to conclude that all differences seen between groups after Western blot analysis are inherent to learning processes during the task and not due to motivational, sensory, or locomotor aspects of the task (Bontempi et al. 1999; Poirier et al. 2008). These yoked controls provide more specific controls than traditional home-cage controls that are left untouched in their cage. Yoked controls do learn about their spatial environment, but their special training (they are not exposed to a choice and are forced to enter an arm, matching or not a previous visit) makes rule learning irrelevant for them.

\section{Low interference working memory (LIWM) task}

Rats trained in the LIWM task $(n=10)$ were submitted to four trials per day, each consisting of a sample and a choice phase (matching the eight runs performed by the RM group-see Fig. 1D). In the sample phase, rats were first allowed to enter from a starting arm one randomly chosen baited arm while all other arms remained closed. Rats were then returned to the transfer cage for a short delay of $15 \mathrm{sec}$ (identical delay and procedure than in the RM task). During the subsequent choice phase, rats were presented with two adjacent arms, the familiar arm that had just been visited and empty of food, and an adjacent arm containing a second food reward. Rats had to choose the novel arm to be positively reinforced (classical delayed-non-match-to-place task). Different pairs of arms were used for each trial. Like for RM rats, each experimental LIWM rat was paired with a yoked control (YLIWM) that was exposed to the same radial maze arms to make sure that the two groups (LIWM and YLIWM) were exposed to the same spatial information. Whereas LIWM rats had to learn a delayed-nonmatch-to-place task rule to successfully complete the task, YLIWM rats $(n=10)$ were exposed to an equal number of nonmatch and match trials in a pseudorandom fashion to prevent YLIWM rats to predict the outcome of a trial. However, like YRM rats, YLIWM rats were forced to visit only one arm during each run and in consequence were not exposed to any cognitive choice when compared with LIWM rats. 


\section{High interference working memory (HIWM) task}

HIWM rats $(n=12)$ were exposed to an experimental procedure similar to the one used in the LIWM task, except that the same pair of arms was used every day for each trial. This procedure promoted high level of interference and repetition to make forgetting of previous trials necessary to complete an ongoing trial (Fig. 1C; Roberts and Dale 1981; Saxe et al. 2007; Malleret et al. 2010). Each experimental HIWM rat was paired with a yoked control (YHIWM; $n=12$ ) that performed the same amount of motor activity and ate the same number of pellets as already described for the YLIWM group.

\section{Western blot}

After the last trial on the last day of training, rats were immediately decapitated and their brains were rapidly removed on a bed of dry ice. Brains were immediately plunged into isopentane at $-50^{\circ} \mathrm{C}$ and were soaked for $10-15 \mathrm{~min}$. All brains were stored at $-80^{\circ} \mathrm{C}$. Each brain was individually dissected with a Cryostat (Microm HM550) kept at $-14^{\circ} \mathrm{C}$. Of note, $300-\mu \mathrm{m}$ thick sections containing the medial Prefrontal Cortex (mainly prelimbic area), and the dorsal hippocampus (dCA1, dCA3, and the DG) were sliced and structures were micropunched under microscope guidance by using small trocars adapted to the size of these structures. Overall protein concentrations were obtained using the Bradford method (Bradford 1976). Briefly, using precise concentrations of bovine serum albumin (BSA), samples with known protein concentrations were prepared and scanned using a spectrophotometer $(\lambda=595 \mathrm{~nm})$ to establish a standard curve. A given sample was combined with homogenization buffer and Bradford Reagent $(4.5 \%$ Coomassie Blue G250, 10\% orthophosphoric acid). Samples were scanned using the spectrophotometer and protein concentration readings were recorded. Samples were then individually diluted with homogenization buffer and were denatured at $65^{\circ} \mathrm{C}$ during $5 \mathrm{~min}$ in denaturation buffer $(125$ $\mathrm{mM}$ Tris pH 6.8, $50 \mathrm{mM}$ dithiothreitol (DTT), 1\% sodium dodecylsulfate (SDS), $0.005 \%$ bromophenol blue, $8 \%$ glycerol) to contain a final protein concentration of $10 \mu \mathrm{g} / 10 \mu \mathrm{L}$. Denatured samples were aliquoted and stored at $-80^{\circ} \mathrm{C}$ until further analysis. Each sample was then deposited on an electrophoresis precast gel (4\%-12\% tris-bis-SDS PAGE Bio-Rad). Gels were run at constant voltage $80 \mathrm{~V}$ for $15 \mathrm{~min}$ to compress the bands of protein and then run at $110 \mathrm{~V}$ for $90 \mathrm{~min}$ to separate the proteins according to their size. Gels were then cut into three bands each containing a group of relevant protein (250-150 kDa for the NMDAR; $150-80$ $\mathrm{kDa}$ for the AMPAR; and 80-30 kDa for $\beta$-Tubulin and CaMKII) as described in Kiyatkin and Aksamitiene (2009). These bands were then deposited on a nitrocellulose membrane (Whatman) and transferred (Criterion Blotter, Bio-Rad) at $100 \mathrm{~V}$ for $40 \mathrm{~min}$ at $4^{\circ} \mathrm{C}$. Once the transfer was completed, membranes were soaked in Red Ponceau to verify the good transfer of protein from the gel to the membrane. Membranes were blocked in TBS (Tris buffer saline) $+5 \%$ milk for at least $1 \mathrm{~h}$ under agitation before exposure to antibodies. Membranes were then cut and incubated in primary antibodies anti-phosphorylated $\alpha$-CAMKII at the tyrosine 286 site (tebu-bio, 1:100), anti-CaMKII (Abcam, 1:6000), anti-glutamate AMPA receptor 1 phosphorylated at the serine 831 (ser831) site (Millipore, 1:500), anti-glutamate AMPA receptor 1 phosphorylated at the serine 845 (ser845) site (Millipore, 1:500), antiglutamate AMPA receptor 1 (Millipore, 1:10,000), anti-glutamate NMDA receptor 1 (Millipore, 1:500), anti-glutamate NMDA receptor 2A (Millipore, 1:4000), anti-glutamate NMDA receptor 2B (Millipore, 1:1000) or anti- $\beta$-Tubulin III (Sigma-Aldrich, 1:2000), in TBS-T (Tris-buffered saline-0.1\% Tween 20) $+3 \%$ milk overnight at $4^{\circ} \mathrm{C}$ under agitation. The next morning membranes were removed from the primary antibody solution and were washed for $3 \times 10 \mathrm{~min}(2 \times$ TBS-T $1 \times$ TBS $)$ before incubation for 120 min in secondary antibody (in TBS $+3 \%$ milk) all at $4^{\circ} \mathrm{C}$ and under agitation. Following secondary antibody incubation, membranes were washed for $3 \times 10 \mathrm{~min}(2 \times \mathrm{TBS}-\mathrm{T} 1 \times \mathrm{TBS})$ under agitation and were then exposed to fluorescent ECL substrate (Epirubicin-Cisplatin-5-Fluoro-uracile) to cause a fluorescent reaction between the secondary antibody and the ECL. Band fluo- rescence was captured by a FluorImager (Molecular Dynamics). After revelation, membranes were reused using a stripping solution for $90 \mathrm{~min}$ and washed for $3 \times 10 \mathrm{~min}(2 \times$ TBS-T $1 \times$ TBS $)$ before blocking and antibodies exposure.

\section{Statistical analysis}

\section{Behavior}

After checking for normality and homogeneity of variances, behavioral data were analyzed using two-way ANOVAs (analysis of variance) for repeated measures with Block (corresponding to two consecutive days of training) and Group (RM, LIWM, HIWM) as main factors (Statview 5.0.). Further comparisons were performed by post hoc (Scheffe and split-by Group) analyses for particular within-group comparisons. Data are expressed as means of performances \pm S.E.M.

\section{Western blot}

Western blot analysis was done with Image $(\mathrm{NIH})$ using the gel analysis plug-in. Each band was normalized to its corresponding loading control band: the housekeeping protein $\beta$-Tubulin. As no significant statistical difference in any proteins expression and in any of the structures was found between the three control groups (YLIWM, YHIWM, and YRM), they were pooled as one single control group. The ratio protein of interest/ $\beta$-tubulin was calculated for each duplicate and for each protein of interest. The quality of each step was controlled by running each experiment twice. When values for the two duplicates varied for $>25 \%$, the sample was not taken into account into the statistical analysis (see final number of validated samples for each group and protein in Supplemental Table 1). Each animal average value for the two duplicates was then expressed as a percentage of the control group mean. Statistical results were obtained using StatView 5.0. After checking for normality and homogeneity of variances, nonparametric Kruskal-Wallis tests were performed to analyze Western blot results. Post hoc Mann-Whitney $U$-tests combined with Holm-Bonferroni's corrections for multiple comparisons were used to identify significant differences between groups. Data are expressed as mean of normalized ratio (percentage of control) \pm S.E.M.

\section{Correlations}

Linear regression analysis was performed to assess correlation between CaMKII phosphorylation ratio and GluA1 phosphorylation. We used the Spearman's rank correlation coefficient, a measure of statistical dependencies between nonparametric variables. A positive correlation coefficient between two phosphorylation states indicates that an increase in phosphorylation of one protein (i.e., PCaMKII/CaMKII) results in a proportional increase in the phosphorylation of the other protein (i.e., Ser831/GluA1).

\section{Acknowledgments}

This research was supported by grants from CNRS (ATIP program), Fondation pour la recherche sur le cerveau (FRC), and Région Rhône-Alpes (CIBLE program). M.A.J. and N.F. were also supported by Fondation pour la recherche médicale (FRMFDT20130928087) and Région Rhône-Alpes (ARC2 doctoral fellowship). We thank Fernand Malleret for his design and realization of the radial maze apparatus.

\section{References}

Anderson MC, Neely JH, Bjork EL, Bjork RA. 1996. Chapter 8-interference and inhibition in memory retrieval. In Memory, pp. 237-313. Academic Press, San Diego.

Baddeley A. 1981. The concept of working memory: a view of its current state and probable future development. Cognition 10: 17-23.

Barria A, Derkach V, Soderling T. 1997a. Identification of the $\mathrm{Ca}^{2+}$ / calmodulin-dependent protein kinase II regulatory phosphorylation 
site in the $\alpha$-amino-3-hydroxyl-5-methyl-4-isoxazole-propionate-type glutamate receptor. J Biol Chem 272: 32727-32730.

Barria A, Muller D, Derkach V, Griffith LC, Soderling TR. 1997b. Regulatory phosphorylation of AMPA-type glutamate receptors by CaM-KII during long-term potentiation. Science 276: 2042-2045.

Bear MF, Abraham WC. 1996. Long-term depression in hippocampus. Аnnu Rev Neurosci 19: 437-462.

Bear MF, Malenka RC. 1994. Synaptic plasticity: LTP and LTD. Curr Opin Neurobiol 4: 389-399.

Bliss TV, Collingridge GL. 1993. A synaptic model of memory: long-term potentiation in the hippocampus. Nature 361: 31-39.

Bliss TV, Lomo T. 1973. Long-lasting potentiation of synaptic transmission in the dentate area of the anaesthetized rabbit following stimulation of the perforant path. J Physiol 232: 331-356.

Bontempi B, Laurent-Demir C, Destrade C, Jaffard R. 1999. Time-dependent reorganization of brain circuitry underlying long-term memory storage. Nature 400: 671-675.

Botreau F, El Massioui N, Cheruel F, Gisquet-Verrier P. 2004. Effects of medial prefrontal cortex and dorsal striatum lesions on retrieval processes in rats. Neuroscience 129: 539-553.

Bradford MM. 1976. A rapid and sensitive method for the quantitation of microgram quantities of protein utilizing the principle of protein-dye binding. Anal Biochem 72: 248-254.

Braunewell KH, Manahan-Vaughan D. 2001. Long-term depression: a cellular basis for learning? Rev Neurosci 12: 121-140.

Cao X, Wang H, Mei B, An S, Yin L, Wang LP, Tsien JZ. 2008. Inducible and selective erasure of memories in the mouse brain via chemical-genetic manipulation. Neuron 60: $353-366$.

Cowan N. 2008. What are the differences between long-term, short-term, and working memory? Prog Brain Res 169: 323-338.

Delaney PF, Sahakyan L. 2007. Unexpected costs of high working memory capacity following directed forgetting and contextual change manipulations. Mem Cognit 35: 1074-1082.

Dillon GM, Qu XL, Marcus JN, DodartJC. 2008. Excitotoxic lesions restricted to the dorsal CA1 field of the hippocampus impair spatial memory and extinction learning in C57BL/6 mice. Neurobiol Learn Mem 90: 426-433.

Dong Z, Han H, Li H, Bai Y, Wang W, Tu M, Peng Y, Zhou L, He W, Wu X, et al. 2015. Long-term potentiation decay and memory loss are mediated by AMPAR endocytosis. J Clin Invest 125: 234-247.

Dudchenko PA. 2004. An overview of the tasks used to test working memory in rodents. Neurosci Biobehav Rev 28: 699-709.

Dudek SM, Bear MF. 1992. Homosynaptic long-term depression in area CA1 of hippocampus and effects of N-Methyl-D-Aspartate receptor blockade. Proc Natl Acad Sci 89: 4363-4367.

Elgersma Y, Silva AJ. 1999. Molecular mechanisms of synaptic plasticity and memory. Curr Opin Neurobiol 9: 209-213.

Etkin A, Alarcon JM, Weisberg SP, Touzani K, Huang YY, Nordheim A, Kandel ER. 2006. A role in learning for SRF: deletion in the adult forebrain disrupts LTD and the formation of an immediate memory of a novel context. Neuron 50: 127-143.

Fernandez-Monreal M, Brown TC, Royo M, Esteban JA. 2012. The balance between receptor recycling and trafficking toward lysosomes determines synaptic strength during long-term depression. J Neurosci 32: $13200-13205$.

Fukunaga K, Muller D, Miyamoto E. 1995. Increased phosphorylation of $\mathrm{Ca}^{2+}$ /calmodulin-dependent protein kinase II and its endogenous substrates in the induction of long-term potentiation. J Biol Chem 270: 6119-6124.

Giese KP, Fedorov NB, Filipkowski RK, Silva AJ. 1998. Autophosphorylation at Thr286 of the $\alpha$ calcium-calmodulin kinase II in LTP and learning. Science 279: 870-873.

Gold AE, Kesner RP. 2005. The role of the CA3 subregion of the dorsal hippocampus in spatial pattern completion in the rat. Hippocampus 15: 808-814.

Herron CE, Lester RA, Coan EJ, Collingridge GL. 1986. Frequencydependent involvement of NMDA receptors in the hippocampus: a novel synaptic mechanism. Nature 322: 265-268.

Joseph MA, Fraize N, Ansoud-Lerouge J, Sapin E, Peyron C, Arthaud S, Libourel PA, Parmentier R, Salin PA, Malleret G. 2015. Differential involvement of the dentate gyrus in adaptive forgetting in the rat. PLoS One 10: e0142065.

Kandel ER. 2001. The molecular biology of memory storage: a dialogue between genes and synapses. Science 294: 1030-1038.

Kesner RP, Churchwell JC. 2011. An analysis of rat prefrontal cortex in mediating executive function. Neurobiol Learn Mem 96: 417-431.

Kesner RP, Rolls ET. 2001. Role of long-term synaptic modification in short-term memory. Hippocampus 11: 240-250.

Kesner RP, Rolls ET. 2015. A computational theory of hippocampal function, and tests of the theory: new developments. Neurosci Biobehav Rev 48: 92-147.

Kiyatkin A, Aksamitiene E. 2009. Multistrip western blotting to increase quantitative data output. Methods Mol Biol 536: 149-161.
Lee HK, Barbarosie M, Kameyama K, Bear MF, Huganir RL. 2000. Regulation of distinct AMPA receptor phosphorylation sites during bidirectional synaptic plasticity. Nature 405: 955-959.

Levy BJ, Kuhl BA, Wagner AD. 2010. The functional neuroimaging of forgetting. In Forgetting (ed. Della Sala S), pp. 135-163. Psychology Press, New York.

Lisman J, Yasuda R, Raghavachari S. 2012. Mechanisms of CaMKII action in long-term potentiation. Nat Rev Neurosci 13: 169-182.

Malleret G, Haditsch U, Genoux D, Jones MW, Bliss TV, Vanhoose AM, Weitlauf C, Kandel ER, Winder DG, Mansuy IM. 2001. Inducible and reversible enhancement of learning, memory, and long-term potentiation by genetic inhibition of calcineurin. Cell 104: 675-686.

Malleret G, Alarcon JM, Martel G, Takizawa S, Vronskaya S, Yin D, Chen IZ, Kandel ER, Shumyatsky GP. 2010. Bidirectional regulation of hippocampal long-term synaptic plasticity and its influence on opposing forms of memory. J Neurosci 30: 3813-3825.

Martin SJ, Morris RG. 2002. New life in an old idea: the synaptic plasticity and memory hypothesis revisited. Hippocampus 12: 609-636.

Maviel T, Durkin TP, Menzaghi F, Bontempi B. 2004. Sites of neocortical reorganization critical for remote spatial memory. Science 305: 96-99.

Miller SG, Kennedy MB. 1986. Regulation of brain type II Ca ${ }^{2+}$ calmodulin-dependent protein kinase by autophosphorylation: a $\mathrm{Ca}^{2+}$-triggered molecular switch. Cell 44: 861-870.

Nayak A, Zastrow DJ, Lickteig R, Zahniser NR, Browning MD. 1998. Maintenance of late-phase LTP is accompanied by PKA-dependent increase in AMPA receptor synthesis. Nature 394: 680-683.

Nicholls RE, Alarcon JM, Malleret G, Carroll RC, Grody M, Vronskaya S, Kandel ER. 2008. Transgenic mice lacking NMDAR-dependent LTD exhibit deficits in behavioral flexibility. Neuron 58: 104-117.

Okuno H, Akashi K, Ishii Y, Yagishita-Kyo N, Suzuki K, Nonaka M, Kawashima T, Fujii H, Takemoto-Kimura S, Abe M, et al. 2012. Inverse synaptic tagging of inactive synapses via dynamic interaction of Arc/ Arg3.1 with CaMKIIß. Cell 149: 886-898.

Poirier GL, Amin E, Aggleton JP. 2008. Qualitatively different hippocampal subfield engagement emerges with mastery of a spatial memory task by rats. J Neurosci 28: 1034-1045.

Rauchs G, Feyers D, Landeau B, Bastin C, Luxen A, Maquet P, Collette F. 2011. Sleep contributes to the strengthening of some memories over others, depending on hippocampal activity at learning. J Neurosci 31: $2563-2568$

Reisel D, Bannerman DM, Schmitt WB, Deacon RM, Flint J, Borchardt T, Seeburg PH, Rawlins JN. 2002. Spatial memory dissociations in mice lacking GluR1. Nat Neurosci 5: 868-873.

Roberts WA, Dale RHI. 1981. Remembrance of places lasts: proactive inhibition and patterns of choice in rat spatial memory. Learn Motiv 12: 261-281.

Rosenzweig ES, Barnes CA, McNaughton BL. 2002. Making room for new memories. Nat Neurosci 5: 6-8.

Sala SD. 2010. Forgetting. Taylor \& Francis.

Sanderson DJ, Good MA, Skelton K, Sprengel R, Seeburg PH, Rawlins JN, Bannerman DM. 2009. Enhanced long-term and impaired short-term spatial memory in GluA1 AMPA receptor subunit knockout mice: evidence for a dual-process memory model. Learn Mem 16: 379-386.

Sanhueza M, McIntyre CC, Lisman JE. 2007. Reversal of synaptic memory by $\mathrm{Ca}^{2+} /$ calmodulin-dependent protein kinase II inhibitor. J Neurosci 27: $5190-5199$.

Saxe MD, Malleret G, Vronskaya S, Mendez I, Garcia AD, Sofroniew MV, Kandel ER, Hen R. 2007. Paradoxical influence of hippocampal neurogenesis on working memory. Proc Natl Acad Sci 104: 4642-4646.

Schmitt WB, Deacon RM, Seeburg PH, Rawlins JN, Bannerman DM. 2003. A within-subjects, within-task demonstration of intact spatial reference memory and impaired spatial working memory in glutamate receptor-A-deficient mice. J Neurosci 23: 3953-3959.

Schmitt WB, Sprengel R, Mack V, Draft RW, Seeburg PH, Deacon RM, Rawlins JN, Bannerman DM. 2005. Restoration of spatial working memory by genetic rescue of GluR-A-deficient mice. Nat Neurosci 8: $270-272$.

Shi SH, Hayashi Y, Petralia RS, Zaman SH, Wenthold RJ, Svoboda K, Malinow R. 1999. Rapid spine delivery and redistribution of AMPA receptors after synaptic NMDA receptor activation. Science 284: 1811-1816.

Tononi G, Cirelli C. 2003. Sleep and synaptic homeostasis: a hypothesis. Brain Res Bull 62: 143-150.

Wang H, Shimizu E, Tang YP, Cho M, Kyin M, Zuo W, Robinson DA, Alaimo PJ, Zhang C, Morimoto H, et al. 2003. Inducible protein knockout reveals temporal requirement of CaMKII reactivation for memory consolidation in the brain. Proc Natl Acad Sci 100: 4287-4292.

Whitlock JR, Heynen AJ, Shuler MG, Bear MF. 2006. Learning induces long-term potentiation in the hippocampus. Science 313: 1093-1097.

Received July 18, 2016; accepted in revised form November 23, 2016. 


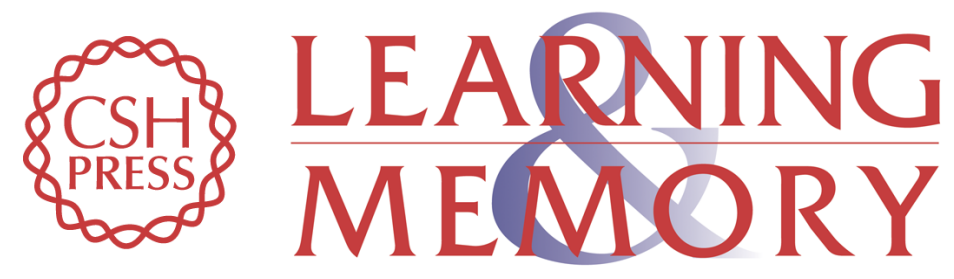

\section{Differential changes in hippocampal CaMKII and GluA1 activity after memory training involving different levels of adaptive forgetting}

Nicolas Fraize, Al Mahdy Hamieh, Mickaël Antoine Joseph, et al.

Learn. Mem. 2017, 24:

Access the most recent version at doi:10.1101/Im.043505.116

\section{Supplemental http://learnmem.cshlp.org/content/suppl/2017/01/11/24.2.86.DC1 Material}

References This article cites 55 articles, 18 of which can be accessed free at: http://learnmem.cshlp.org/content/24/2/86.full.html\#ref-list-1

Creative This article is distributed exclusively by Cold Spring Harbor Laboratory Press for the Commons first 12 months after the full-issue publication date (see

License http://learnmem.cshlp.org/site/misc/terms.xhtml). After 12 months, it is available under a Creative Commons License (Attribution-NonCommercial 4.0 International), as described at http://creativecommons.org/licenses/by-nc/4.0/.

Email Alerting Receive free email alerts when new articles cite this article - sign up in the box at the Service top right corner of the article or click here. 